\title{
On the Stability of Linear Systems
}

\author{
by Daniele Sasso *
}

\begin{abstract}
The criteria of stability defined in the standard theory of linear systems aren't exhaustive and show some inconsistencies. In this article we define new criteria of stability more consistent with real physical situations. In particular we distinguish between static stability and dynamic stability in order to analyse the stability of systems in the time domain and in Laplace's equivalent domain. Let introduce then the frequency stability in order to analyse the stability of systems in the Fourier domain.
\end{abstract}

\section{Mathematical models of linear systems}

A physical system is linear if it respects the principle of superposition of effects and it is stationary if its functioning is always the same at any time. The mathematical model for representing a linear physical system is given in the time domain by the linear differential equation

$a_{n} \frac{d y^{n}(t)}{d t^{n}}+a_{n-1} \frac{d y^{n-1}(t)}{d t^{n-1}}+\ldots+a_{1} \frac{d y(t)}{d t}+a_{0} y(t)=b_{m} \frac{d x^{m}(t)}{d t^{m}}+b_{m-1} \frac{d x^{m-1} x(t)}{d t^{-t}}+\ldots .+b_{1} \frac{d x(t)}{d t}+b_{0} x(t)$

where $t$ is the time variable, $x(t)$ is the input function, $y(t)$ is the output function, $a_{i}$ and $b_{j}$ are real numbers. If $a_{i}(i=1,2, \ldots . n)$ and $b_{j}(j=1,2, \ldots . m)$ are constant the linear system is also stationary.

In Laplace's domain the linear system is represented by the algebraic equation

$a_{n} s^{n} Y(s)+a_{n-1} s^{n-1} Y(s)+\ldots .+a_{1} s Y(s)+a_{0} Y(s)=b_{m} s^{m} X(s)+b_{m-1} s^{m-1} X(s)+\ldots+b_{1} s X(s)+b_{0} X(s)$

where $s=\alpha+j \omega$ is Laplace's complex variable, $Y(s)$ and $X(s)$ are Laplace's transforms of $y(t)$ and $x(t)$. The equation (2) is valid when all the initial conditions whether for the input function and its derivatives or the output function and its derivatives are null.

The transfer function $\mathrm{G}(\mathrm{s})$ (fig.1) is the ratio between output function and input function and from (2) we have

$$
G(s)=\frac{Y(s)}{X(s)}=\frac{b_{m} s^{m}+b_{m-1} s^{m-1}+\ldots+b_{1} s+b_{0}}{a_{n} s^{n}+a_{n-1} s^{n-1}+\ldots .+a_{1} s+a_{0}}
$$

The $\mathrm{m}$ roots of the algebraic numerator are the zeroes of the system and the $\mathrm{n}$ roots of the algebraic denominator are the poles of the system.

* e_mail: dgsasso@alice.it 
The general criterion of stability for a stationary and linear physical system ${ }^{[1]}$ affirms that

"a physical system is stable only if the poles of the system are negative real or have negative real part if the pole is complex."

It suffices only one positive real pole or one complex pole with positive real part so that the system is unstable. It suffices only one null real pole or one complex pole with null real part (both with one degree) so that the system is on the verge of stability. One null real pole or with null real part if complex and with degree greater than one generates always instability in the system.
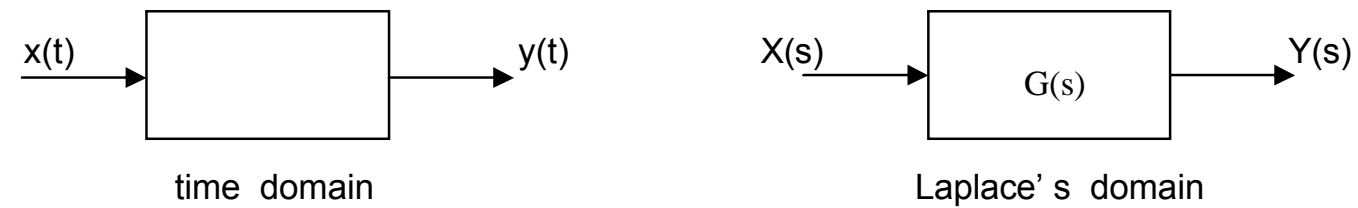

Fig.1 Representation of the system in the time domain and in Laplace's domain.

The general criterion of stability can generate confusion when the input function is sinusoidal or periodic. In that case in fact the general criterion isn' $t$ exhaustive and the system must be analysed in the Fourier domain which is the frequency domain. In fact we will prove that systems can be:

a. stable in time and unstable in frequency

b. stable in frequency and unstable in time

c. stable in time and in frequency

d. unstable in time and in frequency

e. stable for a few frequencies and unstable for other frequencies.

\section{Definition of stability}

The stability of systems in Laplace's domain is equivalent to the stability of systems in the time domain. In order to study the stability of systems we make use of a trial signal like input function (input signal). We distinguish three types of stability ${ }^{[2]}$ :
a. static stability
b. dynamic stability
c. frequency stability

a. static stability

Suppose that the system is initially at rest, i.e. without input signal, and with the output function (output signal) characterized by a constant initial value, for instance null. The trial signal is in that case the Dirac impulse $\delta(t)$ (fig.2). 


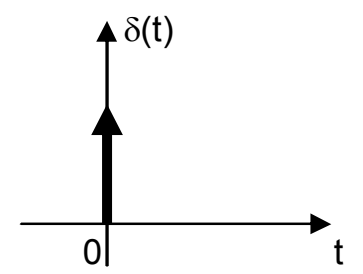

Fig.2 The Dirac impulse is an always null signal unless in the application instant where it is theoretically infinite.

After the application of the trial signal the system has an evolution characterized by a transient phase. The following definition of static stability is valid:

The system is endowed with static stability if after the end of the transient phase it comes back to the initial conditions.

b. dynamic stability

The system is still initially at rest with null initial condition for the output signal. The trial signal in that case is the unitary step signal 1(t) (fig.3). This signal is null for $t<0$ and equals 1 for $t \geq 0$. Therefore the unitary step signal is always limited otherwise than the Dirac signal.

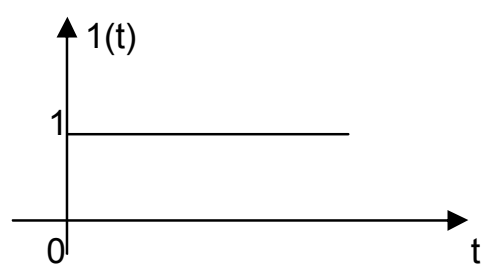

Fig.3 Graphic representation of the unitary step signal.

For the dynamic stability the following definition is valid:

The system is endowed with dynamic stability if also the output signal has always limited amplitude.

c. frequency stability

The system is initially at rest and in that case the sinusoidal signal is used like trial signal. The following definition of frequency stability is valid:

The system is endowed with frequency stability if for all the values of frequency the frequency answer of the system is always limited.

The frequency answer is the amplitude of the permanent answer of the output signal at the end of the transient phase.

It is possible to observe that the trial signal changes in the three physical conditions of stability. For the static stability the impulsive signal needs using, 
for both the dynamic stability and the frequency stability a limited and permanent signal needs using. For the frequency stability a signal with contents of frequency needs also using.

We can now to analyse the stability of systems enunciating a few stability criteria.

\section{Stability criteria}

Let us consider a stationary and linear system whose transfer function in Laplace's domain is

$$
G(s)=\frac{k\left(s-z_{1}\right)\left(s-z_{2}\right) \ldots \ldots \ldots\left(s-z_{m}\right)}{\left(s-p_{1}\right)\left(s-p_{2}\right) \ldots \ldots \ldots\left(s-p_{n}\right)}
$$

where $z_{j}(j=1,2, \ldots m)$ are the zeroes of the system, $p_{i}(i=1,2, \ldots . n)$ are the poles and $k=b_{m} / a_{n}$.

In Fourier's domain we have

$$
\begin{gathered}
G(j \omega)=\frac{k\left(j \omega-z_{1}\right)\left(j \omega-z_{2}\right) \ldots \ldots \ldots\left(j \omega-z_{m}\right)}{\left(j \omega-p_{1}\right)\left(j \omega-p_{2}\right) \ldots \ldots \ldots\left(j \omega-p_{n}\right)} \\
Y(j \omega)=G(j \omega) X(j \omega) \\
\omega=2 \pi f
\end{gathered}
$$

\subsection{Static stability}

In order to study the static stability the Dirac signal must be applied like input to the system supposed initially at rest. In that case we have

$$
Y(s)=G(s) X(s)=\frac{k\left(s-z_{1}\right)\left(s-z_{2}\right) \ldots \ldots \ldots\left(s-z_{m}\right)}{\left(s-p_{1}\right)\left(s-p_{2}\right) \ldots \ldots \ldots\left(s-p_{n}\right)} X(s)
$$

Because for the Dirac signal $X(s)=1$

$$
Y(s)=\frac{k\left(s-z_{1}\right)\left(s-z_{2}\right) \ldots \ldots \ldots\left(s-z_{m}\right)}{\left(s-p_{1}\right)\left(s-p_{2}\right) \ldots \ldots \ldots\left(s-p_{n}\right)}
$$

Making use of the algebraic factorization we have

$$
Y(s)=\frac{A_{1}}{s-p_{1}}+\frac{A_{2}}{s-p_{2}}+\ldots \ldots+\frac{A_{n}}{s-p_{n}}
$$

and passing from Laplace's domain to the time domain through Laplace's antitransform we have

$$
y(t)=A_{1} e^{p_{1} t}+A_{2} e^{p_{2} t}+\ldots \ldots+A_{n} e^{p_{n} t}
$$


Every term $A_{i} e^{p_{i t}}(i=1,2, \ldots . n)$ can have a different evolution according as the pole $p_{i}$ is real or complex. If the pole $p_{i}$ is real it can be positive, null or negative and the three possible evolutions are represented in fig.4.

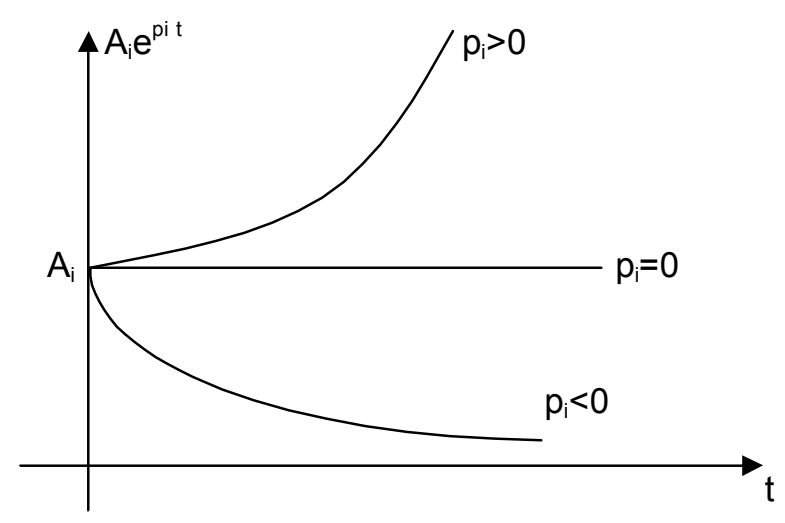

Fig.4 The evolutions of the term $A_{i} e^{\text {pit }}$ when the pole $p_{i}$ is real.

If the pole $p_{i}$ is complex certainly there is also the conjugate pole $p_{i}^{*}$ because the complex algebraic roots are always in pair. In that case we have

$$
\begin{aligned}
& p_{i}=\alpha_{i}+j \omega_{i} \\
& p_{i}^{*}=\alpha_{i}-j \omega_{i}
\end{aligned}
$$

and making use of Eulero's formulas

$$
y_{i i^{*}}(t)=A_{i} e^{p_{i} t}+A_{i}^{*} e^{p_{i t} t}=2 A e^{\alpha_{i} t} \cos \omega_{i} t
$$

where $A_{i}=A_{i}^{*}=A$.

According as the real part $\alpha_{i}$ is positive, negative o null the couple of complex poles can have three possible evolutions represented in fig.5.

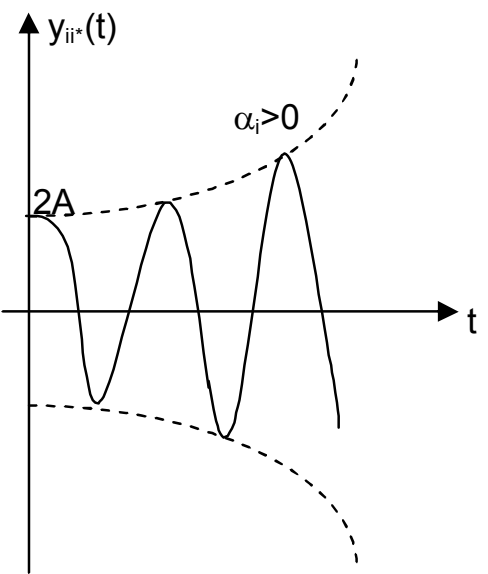

a.

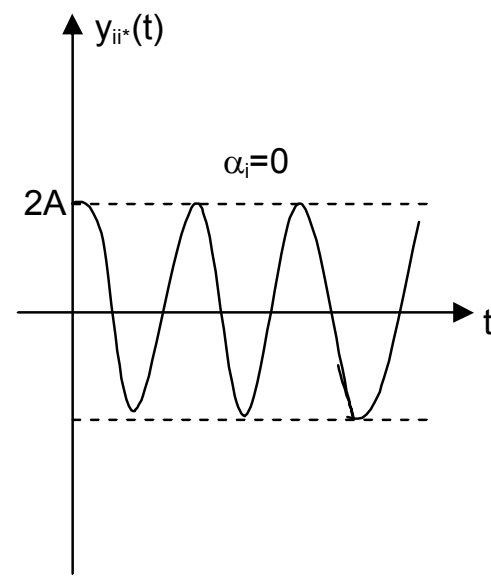

b.

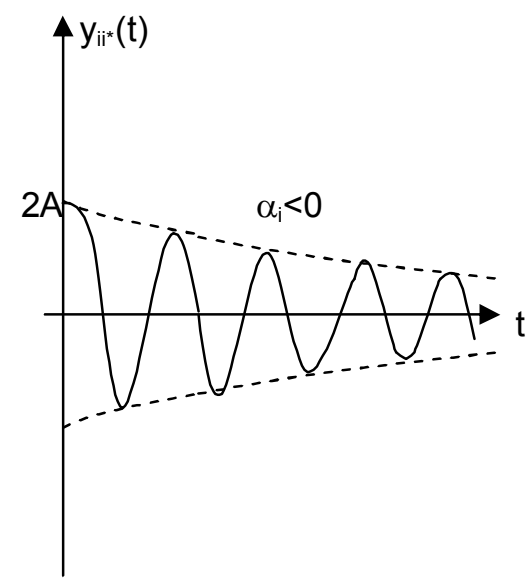

C.

Fig.5 Evolution of the term $\mathrm{y}_{\mathrm{ii}^{*}}(\mathrm{t})$ due to one couple of complex poles
a. the real part $\alpha_{i}$ is positive
b. the real part $\alpha_{i}$ is null
c. the real part $\alpha_{i}$ is negative 
As per the given definition of static stability we can enunciate the following criterion of static stability:

The system is endowed with static stability only if the poles of the system are negative real or have negative real part if the pole is complex. If there is one null real pole or one complex pole with null real part (both with one degree) the system is on the verge of stability.

The verge of stability is anyway a bland form of instability. We see also that the criterion of static stability is equivalent to the general criterion of stability.

\subsection{Dynamic stability}

Let us apply in that case the unitary step signal in input to system that is supposed initially at rest. Being $X(s)=1 / s$ the input signal addes a null real pole to the output signal $Y(s)=G(s) X(s)$.

As per the given definition of dynamic stability the following criterion of dynamic stability is valid:

The system is endowed with dynamic stability only if the poles of the system are negative real or have negative real part if the pole is complex.

For the static stability one null real pole or one complex pole with null real part (one degree) generates the verge of stability, for the dynamic stability one null real pole (with any degree) generates always instability while one couple of complex poles with null real part (imaginary poles) doesn't generate instability.

There are therefore a few differences between the static stability and the dynamic stability.

\subsection{Frequency stability}

With reference to (5) and (6) we see that if the input signal is sinusoidal the amplitude of the permanent answer of the output signal is infinite and not limited if one of the following two conditions occurs

a. $m>n$

b. there is an imaginary pole in the transfer function.

In fact if $m>n$ we have instability for very high frequency $(\omega \rightarrow \infty)$

$$
\lim _{\omega \rightarrow \infty} G(j \omega)=\infty
$$

If the transfer function has one imaginary pole $p_{i}=j \omega_{i}$ then the term $j \omega_{i}-p_{i}=0$ and

$$
\lim _{\omega \rightarrow \omega_{i}} G(j \omega)=\infty
$$


In that case the sistem is unstable in frequency because it is unstable for the frequency $\omega=\omega_{\mathrm{i}}$.

We can enunciate the following stability criterion in frequency:

The system is endowed with frequency stability only if $m \leq n$ and there aren't imaginary poles in the transfer function.

We see that the condition $m \leq n$ is in our theory an important condition of frequency stability while in the standard theory this condition doesn't have a clear explanation and seems rather a working hypothesis.

\section{Stability of real systems}

Let us analyse now some practical cases:

a. The system with transfer function $G(s)=k s$ is an active derivator realized by operational amplifier, it is stable whether statically or dynamically but is unstable in frequency for very high frequencies because $m>n$ $(\mathrm{m}=1, \mathrm{n}=0)$.

b. The system with transfer function $G(s)=k / s$ is an active integrator realized by operational amplifier, it is stable in frequency but is unstable whether statically or dynamically because the system has a null real pole with one degree. Statically the system is on the verge of stability.

c. The system with transfer function $G(s)=k /(s-p)$ ( $p$ is a negative real pole) is a passive integrator, it is stable because respects all the three conditions of stability.

d. The system with transfer function $G(s)=k /\left(s-p_{1}\right)\left(s-p_{2}\right)$ is stable if both poles are negative real. If conversely one of the two poles is positive real the system is stable in frequency but is unstable whether statically or dynamically.

Many other systems can be considered and their stability can be analysed completely through the three criteria of stability before defined.

\section{References}

[1] A. Lepschy, A. Ruberti, Theory of stationary and linear systems, Siderea editions, 1967

[2] D. Sasso, Criteria of stability of linear systems, (published on line, 1999, temporarily unavailable) 